УДК 621.382.2

05.00.00 Технические науки

АНАЛИЗ ДИНАМИЧЕСКОГО ДИАПАЗОНА
МИКРОВОЛНОВЫХ УСИЛИТЕЛЕЙ НА
РЕЗОНАНСНО-ТУННЕЛЬНЫХ ДИОДАХ С
УЧЕТОМ АВТОСМЕЩЕНИЯ

Осадчий Евгений Николаевич

к.т.н, доцент, SPIN-код: 4919-6157

email: eosadchiy@sfedu.ru

Южный федеральный университет,

Ростов-на-Дону, Россия

В статье рассматривается влияние автосмещения в резонансно-туннельном диоде (РТД) на динамический диапазон усилителей на данных диодах. Рассматривается усиление на резонансной частоте и влияние высших гармоник не учитывается, так как они не создадут значительного падения напряжения на нелинейном сопротивлении диода.

Предполагается, что усиление будет достаточно большое и тогда характер зависимости коэффициентов усиления от степени регенерации будет одинаков для усилителей «на проход» и на «отражение». Это позволяет рассмотреть задачу для обоих типов усилителей на РТД. Рассмотрены виды амплитудных характеристик, которые возможны для усилителя на РТД. Построены зависимости параметра, определяющего динамический диапазон усилителя от положения рабочей точки для нескольких значений произведения сопротивления по постоянному току цепи питания на величину модуля максимальной отрицательной проводимости туннельного промежутка $R G$ max. Для каждого значения $R G$ max при заданных параметрах можно определить положение рабочей точки, обеспечивающее заданный режим усилителя. Построены зависимости величины динамического диапазона с учетом автосмещения и при отсутствии автосмещения для двух значений степени регенерации. Учитывая автосмещение, были выявлены два возможных вида амплитудных характеристик и границы их существования. Для этих двух типов амплитудных характеристик были получены выражения, определяющие динамический диапазон усилителя

КЛючевЫе слова: РЕЗОНАНСНО-ТУННЕЛЬНЫЙ ДИОД, ВОЛЬТАМПЕРНАЯ ХАРАКТЕРИСТИКА (ВАХ) ДИОДА, ДИНАМИЧЕСКИЙ ДИАПАЗОН, КОЭФФИЦИЕНТ УСИЛЕНИЯ, СТЕПЕНЬ РЕГЕНЕРАЦИИ, РЕЖИМ ПИТАНИЯ УСИЛИТЕЛЯ НА РТД

Doi: 10.21515/1990-4665-133-053
UDC 621.382 .2

Technical sciences

\section{ANALYSIS OF THE DYNAMIC RANGE OF MICROWAVE AMPLIFIERS ON RESONANCE-TUNNEL DIODES CONSIDERING AUTOMOTIVE REPLACEMENT}

Osadchiy Evgeniy Nikolaevich

Cand.Tech.Sci., associate professor

SPIN-code: 4919-6157

Southern Federal University, Rostov-on-Don, Russia

The influence of auto-displacement in a resonancetunnel diode (RTD) on the dynamic range of amplifiers on these diodes is considered in the article. The amplification at resonance frequency is considered and the influence of higher harmonics is not taken into account, since they do not create a significant voltage drop at the nonlinear resistance of the diode. It is assumed that the gain will be sufficiently large, and then the nature of the dependence of the gain factors on the degree of regeneration will be the same for amplifiers "per pass" and "reflection". This allows us to consider the problem for both types of amplifiers on the RTD. The types of amplitude characteristics , which are possible for the RTD amplifier, are considered. The dependences of the parameter determining the dynamic range of the amplifier on the position of the operating point for several values of the product of the resistance on the direct current of the supply circuit are calculated for the value of the modulus of the maximum negative conductivity of the tunnel gap $R G \max$. For each value of $R G \max$ for given parameters, you can determine the position of the operating point, which provides the specified amplifier mode. Dependences of the value of the dynamic range are constructed with allowance for auto-displacement and in the absence of auto-displacement for two values of the degree of regeneration. Taking into account autosplacement, two possible types of amplitude characteristics and boundaries of their existence were revealed. For these two types of amplitude characteristics, expressions were obtained that determine the dynamic range of the amplifier

Keywords: RESONANCE-TUNNEL DIODE, CURRENT-VOLTAGE CHARACTERISTIC OF A DIODE, DYNAMIC RANGE, GAIN FACTOR, DEGREE OF REGENERATION, POWER SUPPLY MODE OF A RTD AMPLIFIER 
В работах [1, 2] рассмотрены вопросы нелинейных свойств усилителей на РТД однако без учета автосмещения, возникающего, как правило, в нелинейных режимах и способного значительно изменить условия работы РТД, когда сопротивление цепи питания приближается по значению к отрицательному сопротивлению диода. Таким образом представляет интерес рассмотреть влияние автосмещения в усилителях на РТД, чему и посвящена данная работа. Если рассматривать усиление на резонансной частоте, то влияние высших гармоник можно не учитывать, так как можно предположить, что они не создадут значительного падения напряжения на нелинейном сопротивлении диода. Можно предположить, что усиление достаточно большое, тогда характер зависимости коэффициентов усиления от степени регенерации будет одинаков для усилителей «на проход» и на «отражение». Это позволит рассмотреть задачу для обоих типов усилителей на РТД.

В линейном приближении коэффициент усиления регенеративного усилителя мощности можно представить следующим выражением

$$
K_{0}=A(1-\theta)^{2}
$$

где $\theta=-i^{\prime} / R e[Y]$ - степень регенерации устройства; $i^{\prime}$ - отрицательная проводимость РТД в рабочей точке; $R e[Y]$ - действительная часть полной проводимости схемы, которая подключена параллельно нелинейному сопротивлению диода; $A$ - слабо зависящий от степени регенерации $\theta$ и определяемый типом усилителя, коэффициент.

Учитывая нелинейность вольтамперной характеристики (BАХ) РТД выражение (1) преобразуется к следующему виду

$$
K=A /[1-\theta \mathrm{F}(\mathrm{U})]^{2},
$$

где $F(U)=1+v U^{2}+\mu U^{4}+\cdots \quad-$ представляет амплитуду переменного напряжения на туннельном промежутке. 
Виды амплитудных характеристик $K=f\left(P_{\text {вх }}\right)$, которые возможны для усилителя на РТД изображены на рисунке 1.

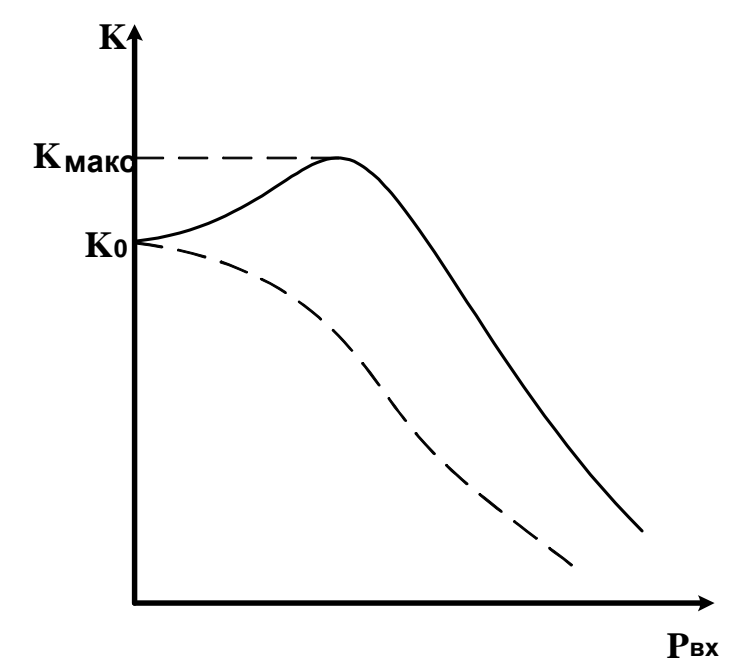

Рисунок 1 - Зависимости $K=f\left(P_{\text {вх }}\right)$

Если $\frac{K_{\text {макс }}}{K_{0}}=n$, где $1 \leq n \leq 2$, то при неравномерности амплитудной характеристики $\frac{K_{\text {макс }}}{K}=2$ выражение для динамического диапазона примет следующий вид

$$
x=-\frac{v}{2 \mu n}\left[1 \pm \sqrt{1-\frac{2 \mu}{v^{2}} \frac{2-n}{n} \frac{1-\theta}{\theta}}\right]
$$

где $x=P_{\mathrm{BX}} K_{0} z_{H} / k$ - определяющий динамический диапазон усилителя параметр; $P_{b x}-$ мощность насыщения, т.е. входная мощность, соответствующая уменьшению коэффициента усиления на 3 децибелла по сравнению с максимальным; $Z_{н}-$ сопротивление нагрузки; $k=U^{2} \mathrm{~B}_{\text {вых }} / U^{2}$ - коэффициент, учитывающий потери мощности на сопротивлении растекания РТД. При частотах намного меньших предельной $k \sim 1$ [3].

Значения параметров $v>0, \mu>0$ и $v<0, \mu>0$ в формуле (3) физически не реализуются, но для случая $v>0, \mu<0$ (формула (3) будет со знаком 
плюс) при $K_{\text {макс }} / K_{0}<81 / 16$ можно реализовать зависимость $K=f\left(P_{\mathrm{Bx}}\right)$, показанную на рисунке 1 сплошной линией, но при нарушении данного неравенства может иметь место амплитудный гистерезис. Найдя максимум правой части выражения (2) и приравнивая его к величине $K_{0} n$ можно получить условие $\frac{K_{\text {макс }}}{K_{0}}=n$. Параметры $v \quad$ и $\mu$ при этом должны удовлетворять условию

$$
\frac{v^{2}}{4 \mu}=\frac{1-\sqrt{n}}{\sqrt{n}} \frac{1-\theta}{\theta}
$$

Подставляя формулу (4) в (3) для $1,5 \leq n \geq 2$ получим выражение, которое определяет $x$ в следующем виде

$$
x \sim \frac{0.586}{v} \frac{1-\theta}{\theta}
$$

С учетом автосмещения величины $v$ и $\mu$ можно определить из $\mathrm{BAX}$ РТД с помощью выражений [4]

$$
\begin{gathered}
v=\frac{1}{8 i^{\prime}}\left[i^{\prime \prime \prime}-\frac{2\left(i^{\prime \prime}\right)^{2}}{\frac{1}{R}+i^{\prime}}\right], \\
\mu=\frac{1}{192 i^{\prime}}\left[i^{(5)}-9 \frac{i^{\prime \prime} i^{(4)}}{\frac{1}{R}+i^{\prime}}+12 \frac{\left(i^{\prime \prime}\right)^{2} i^{\prime \prime}}{\left(\frac{1}{R}+i^{\prime}\right)}\right],
\end{gathered}
$$

где $R$ - сопротивление по постоянному току цепи питания ( $i$ с индексом это дифференцирование $i(u)$ по $u$, но вместо $u$ подставляем $E_{0}$-напряжение смещения). Применяя аппроксимацию ВАХ РТД вида [я]

$$
i=a u e^{-u / U_{1}}+I_{0}\left(e^{D u}-1\right)
$$

где $U_{1}$ - напряжение в максимуме BAX. Если пренебречь диффузионным током и подставив (6) и (7) в формулу (4), то можно получить 


$$
\frac{v^{2}}{4 \mu}=\frac{3}{4} \frac{\left[3-y-2(y-2)^{2} F(y)^{2}\right]}{(1-y)\left[5-y-9(y-2)\left(y-4 F(y)+12(y-2)^{2}(3-y) F^{2}(y)\right]\right.}
$$

где

$$
F(y)=\left[1-y+\frac{1}{R G_{\text {макс }}} e^{y-2}\right]^{-1} ; y=\frac{E_{0}}{U_{1}}
$$

$$
G_{\text {макс }}=a e^{-2}-\text { величина модуля максимальной отрицательной }
$$
проводимости туннельного промежутка.

На рисунке 2 представлены построенные по формуле зависимости $v^{2} / 4 \mu=f\left(E_{0} / U_{1}\right)$ для нескольких значений $R G_{\max }$.

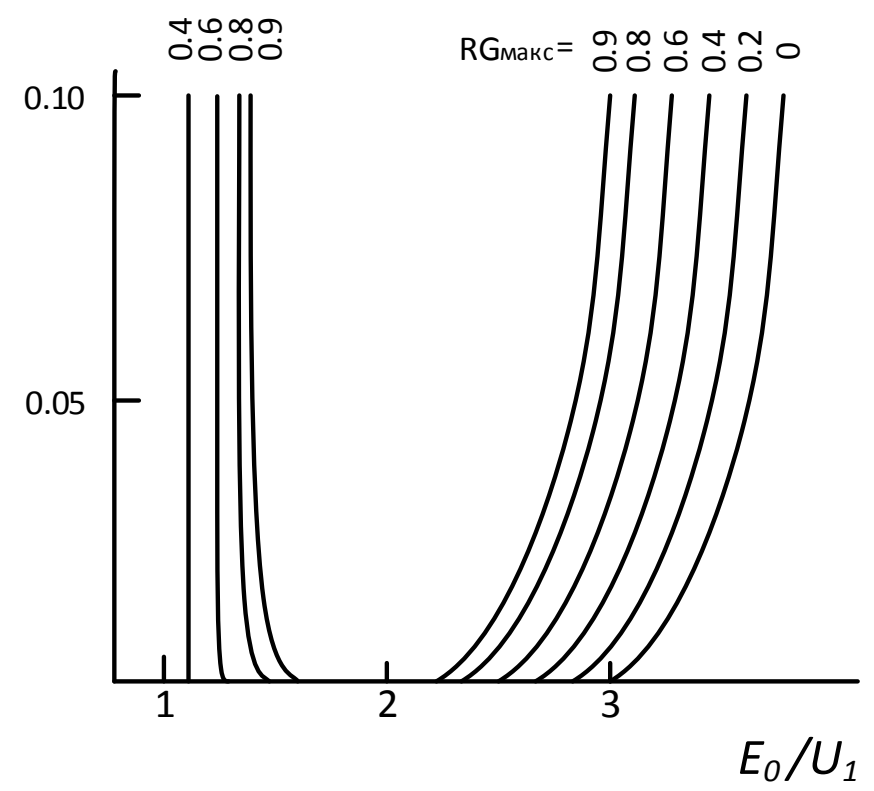

Рисунок 2 - Зависимости $v^{2} / 4 \mu=f\left(E_{0} / U_{1}\right)$

Для каждого значения $R G$ max при заданных $n$ и $\theta$, используя рисунок 2 , можно при помощи формулы (4) определить положение рабочей точки $E_{0} / U_{1}$, обеспечивающее заданный режим усилителя. Определить значение параметра $x$, характеризующего динамический диапазон, можно подставив полученное значение $E_{0} / U_{1}$ в (6) а затем (6) в (5). Показанные сплошной кривой на рисунке 1 , границы существования амплитудной характеристики, определяемой из условия $v=0$, записываются в виде 


$$
R G_{\text {макс }}=\left[\frac{2(y-2)^{2}}{3-y}+y-1\right]^{-1} e^{y-2}
$$

Когда величина $R G_{\text {max }}$ больше значений, определяемой правой частью формулы (10), амплитудные характеристики будут иметь вид сплошных кривых на рисунке 1 . Если $R G_{\text {max }}=1$, что соответствует границе устойчивости схемы по постоянному току, амплитудные характеристики имеют место во всех рабочих точках кроме точки перегиба. В отсутствии автосмещения (при нулевом сопротивлении цепи питания) они будут наблюдаться лишь при значении $E_{0} / U_{1}$ большим трем.

Для стабильной работы (при больших коэффициентах усиления) не всегда требуется возрастание $K_{y}$ с ростом уровня сигнала (пунктир на рисунке 1). В данном случае $v \leq 0, \mu<0$ (в формуле (3) - знак минус, $n=1$ ). Для увеличения динамического диапазона режим питания схемы усилителя подбираем таким образом, чтобы $v$ и $\mu$ имели минимальные значения. Как следует из формулы (10), подбирая величину $R$, при заданном $E_{0}$, параметр $v$ может быть сделан равным нулю на значительной части ВАХ РТД. Динамический диапазон при этом

$$
x=\sqrt{-\frac{1}{2 \mu_{1}} \frac{1-\theta}{\theta}} .
$$

Здесь выражение для $\mu_{1}$ можно определить подставляя (10) в (7) и при использовании аппроксимации (8) оно будет иметь следующий вид

$$
\mu_{1}=-\frac{1}{384 U_{1}^{4}}\left[1+\frac{4 y^{2}-16 y+10}{(1-y)(y-2)^{2}}\right] .
$$

Зависимости $x^{2} / U_{1}^{2}=f\left(E_{0} / U_{1}\right), \quad$ построенные по формуле представлены на рисунке 3 сплошной линией, штрих-пунктирной кривой построенные по формуле (3), когда предполагается отсутствие автосмещения для двух значений степени регенерации. На рисунке 3 
видно, что в области, находящейся правее точки перегиба ВАХ, автосмещение будет вызывать малое уменьшение динамического диапазона $(x)$ при небольших значениях степени регенерации $(\theta=0,8)$ и некоторое его увеличение при больших значениях $\theta \quad(\theta=0,9)$.

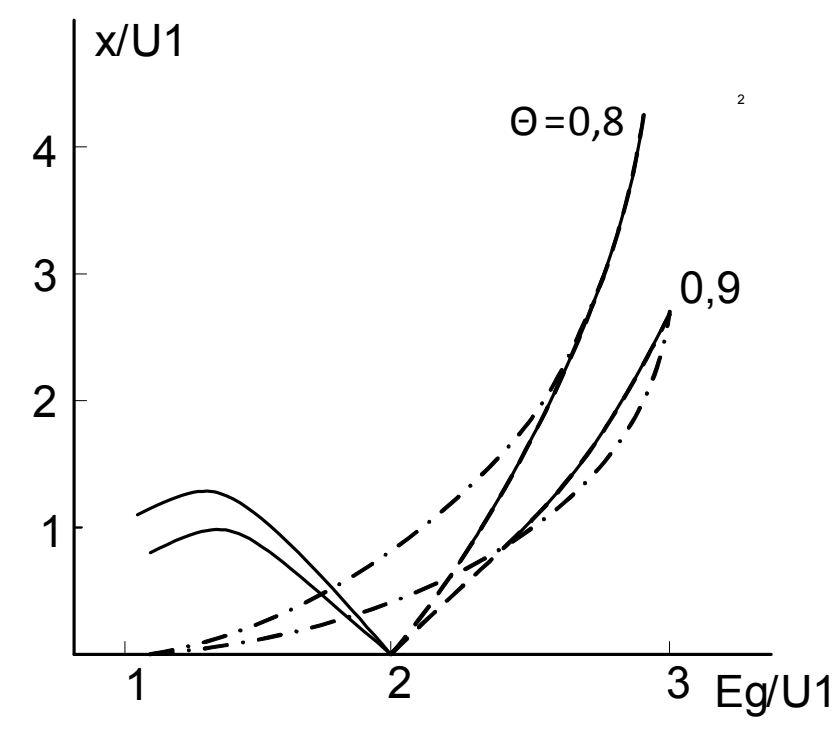

Рисунок 3 - Зависимости $x^{2} / U_{1}^{2}=f\left(E_{0} / U_{1}\right)$

Это увеличение будет тем больше, чем больше величина $\theta$. В области, лежащей левее точки перегиба также подбирая величину $R$ можно добиться некоторого увеличения $x$. Условие $v=0$ в точке $E_{0} / U_{1}=2$ без нарушения устойчивости цепи по постоянному току не может быть выполнено (на рисунке 3 этот участок сплошной линии показан пунктиром). Для амплитудной характеристики, изображенной на рисунке 1 , ширина динамического диапазона будет определяться сопротивлением цепи питания, предельная величина которого будет при $E_{0} / U_{1}=3$ и $R=0$. Значение $E_{0} / U_{1}$, которое соответствует максимальной величине $x$ при заданном $R$ можно выразить из (10), а сама величина $x$ находится из выражения (11).

Исходя из вышеизложенного следует, что при использовании амплитудной характеристики первого типа ( сплошная кривая на рисунке 
1) автосмещение расширяет область существования этой амплитудной характеристики и приводит к уменьшению динамического диапазона $(x)$. При амплитудной характеристики второго типа ( пунктирная кривая на рисунке 1) для больших значений степени регенерации можно подбором параметров цепи питания и режима добиться некоторого расширения динамического диапазона. Данный анализ динамического диапазона усилителей на резонансно-туннельных диодах с учетом автосмещения может быть полезным для проектирования усилителей микроволнового диапазона на РТД, работающих в нелинейных режимах.

\section{Литература}

1. Демьяненко А.В., Алексеев Ю.И., Геворкян А.В. Амплитудно-частотная характеристика (АЧХ) усилителя на лавинно-пролетном диоде в режиме детектирования СВЧ-амплитудно-модулированных оптических колебаний // Инженерный вестник Дона. 2014. № 2. C. URL: ivdon.ru/magazine/archive/n2y2014/250/.

2. Осадчий Е.Н. Квазилинейный анализ работы резонансно-туннельного диода с помощью полиномов различных степеней // Успехи современной науки. 2016. №12. С. $132-138$.

3. Будяков П.С., Белич С.С., Семенищев Е.А., Федосеев С.В., Медведев Д.В., Серебряков А.И. Управляемые избирательные усилители СВЧ диапазона // Инженерный вестник Дона. 2012. № 4. C. URL: ivdon.ru/magazine/archive/n2y2012/250/.

4. Алимов А.П. Змий Б.Ф. Синтез усилителей промежуточной частоты с повышенными показателями качества // Инженерный вестник Дона. 2017. №1. URL: ivdon.ru/magazine/archive/n3y2017/250/.

5. Moskaliuk V. Simplified analytical model of resonant-tunneling diode // Abstracts Proceedings of 32nd International Spring Seminar on Electronics Technology "ISSE 2009". Brno, Czech Republic. 2009. C. 1-5.

6. Zohta Y. Improved optical model for resonant tunneling diode // J. Appl. Phys. 1993. Vol. 74. C. 6996-6998.

7. Schulman J.N. Extension of Tsu-Esaki model for effective mass effects in resonant tunneling / J.N. Schulman // Appl. Phys. Lett. 1998. Vol. 72. P. 2829 - 2831.

8. Qiu Z.J. Experimental verification of origin of plateau-like current-voltage characteristics of resonant tunneling diodes / Z.J. Qiu, Y.S. Gui, S.L. Guo et. al. // Appl. Phys. Lett. 2007. Vol. 4. P. 1961-1963.

9. Алкеев Н. В., Любченко В. E., Velling P., Khorenko E., Prost W., Tegude F. J. Эквивалентная схема резонансно-туннельного диода на основе InGaAs/InAlAs в миллиметровом диапазоне длин волн // Радиотехника и электроника. 2004. Т. 49. № 7. C. $886-892$.

10. Капаев В.В. Высокочастотный отклик и возможности перестраиваемого по частоте терагерцового узкополосного усиления в резонансно-туннельных наноструктурах // ЖЭТФ 2013. Т.143. С. 569. 


\section{References}

1. Dem'janenko A.V., Alekseev Ju.I., Gevorkjan A.V. Amplitudno-chastotnaja harakteristika $(\mathrm{AChH})$ usilitelja na lavinno-proletnom diode v rezhime detektirovanija SVChamplitudno-modulirovannyh opticheskih kolebanij // Inzhenernyj vestnik Dona. 2014. № 2. S. URL: ivdon.ru/magazine/archive/n2y2014/250/.

2. Osadchij E.N. Kvazilinejnyj analiz raboty rezonansno-tunnel'nogo dioda $s$ pomoshh'ju polinomov razlichnyh stepenej // Uspehi sovremennoj nauki. 2016. №12. S. 132 138.

3. Budjakov P.S., Belich S.S., Semenishhev E.A., Fedoseev S.V., Medvedev D.V., Serebrjakov A.I. Upravljaemye izbiratel'nye usiliteli SVCh diapazona // Inzhenernyj vestnik Dona. 2012. № 4. S. URL: ivdon.ru/magazine/archive/n2y2012/250/.

4. Alimov A.P. Zmij B.F. Sintez usilitelej promezhutochnoj chastoty s povyshennymi pokazateljami kachestva // Inzhenernyj vestnik Dona. 2017. №1. URL: ivdon.ru/magazine/archive/n3y2017/250/.

5. Moskaliuk V. Simplified analytical model of resonant-tunneling diode // Abstracts Proceedings of 32nd International Spring Seminar on Electronics Technology "ISSE 2009". Brno, Czech Republic. 2009. S. 1-5.

6. Zohta Y. Improved optical model for resonant tunneling diode // J. Appl. Phys. 1993. Vol. 74. S. 6996-6998.

7. Schulman J.N. Extension of Tsu-Esaki model for effective mass effects in resonant tunneling / J.N. Schulman // Appl. Phys. Lett. 1998. Vol. 72. P. $2829-2831$.

8. Qiu Z.J. Experimental verification of origin of plateau-like current-voltage characteristics of resonant tunneling diodes / Z.J. Qiu, Y.S. Gui, S.L. Guo et. al. // Appl. Phys. Lett. 2007. Vol. 4. P. 1961-1963.

9. Alkeev N. V., Ljubchenko V. E., Velling R., Khorenko E., Prost W., Tegude F. J. Jekvivalentnaja shema rezonansno-tunnel'nogo dioda na osnove InGaAs/InAlAs v millimetrovom diapazone dlin voln // Radiotehnika i jelektronika. 2004. T. 49. № 7. S. 886892.

10. Kapaev V.V. Vysokochastotnyj otklik i vozmozhnosti perestraivaemogo po chastote teragercovogo uzkopolosnogo usilenija $\mathrm{v}$ rezonansno-tunnel'nyh nanostrukturah // ZhJeTF 2013. T.143. S. 569. 